BIOMEDICAL AND BIOSOCIAL ANTHROPOLOGY
$\begin{gathered}\text { Official Journal of the International Academy } \\ \text { of Integrative Anthropology } \\ \text { journal homepage: http://bba-journal.com }\end{gathered}$

\title{
Secretory leukocyte protease inhibitor as a marker of proinflammatory response in children with community-acquired pneumonia
}

Dudnyk V. M., Pasik V. Yu.

National Pirogov Memorial Medical University, Vinnytsya, Ukraine

\section{ARTICLE INFO}

Received: 17 March 2021

Accepted: 20 April 2021

UDC: $616.24-002: 079.6: 153$

CORRESPONDING AUTHOR

e-mail: dudnykvm@gmail.com

Dudnyk V. M.
The study of the secretory leukocyte protease inhibitor (SLPI) level in children with pneumonia is important because it may indicate protease-antiprotease imbalance and predict local regulation of proteolytic activity at different degrees of pneumonia in children. The aim of the study was to assess the level of SLPI as a marker of proinflammatory response in the serum of young children, depending on the severity of pneumonia, including the presence or absence of lesions of the hepatobiliary system. The study investigated the SLPI as a marker of the inflammatory response in children with community-acquired pneumonia, depending on the severity of the disease, lesions of the hepatobiliary system, age and sex. The level of SLPI in the serum was determined by enzyme-linked immunosorbent assay using a diagnostic test system from "IMMUNOTECH" (France). "IBM SPSS Statistica" Version 12 (20) was used for statistical processing of the results. It was found that the course of community-acquired pneumonia is accompanied by an increase in the serum level of SLPI in children in parallel with the severity of the disease. The development of a lesion from the GBS reduces the level of a protease inhibitor in the blood serum of children with pneumonia, which indicates a decrease in the body's defense systems. In children 1-12 months, the level of SLPI is higher than in children 1336 months, which indicates more significant protective capabilities of the body of children in the first year of life. It has been shown that an increase in the level of SLPI correlates with an increase in the level of inflammatory cytokines, which indicates a unidirectional change in the formation of local immunity in response to damage to the lung tissue. At the same time, a negative correlation was found between the level of SLPI and the activities of AST, GGT and CRP ( $r x y=-0.054 ; r x y=-0.215 ; r x y=-0.215$, respectively), which indicates a violation of liver function during the development of an infectious-inflammatory process in the body. Studies of the nature of the effects of cytokines and protease inhibitors (protective factors) on the development and maintenance of inflammatory process in the lungs in young children with community-acquired pneumonia with lesions of the hepatobiliary system may be the basis for predicting its course.

Key words: secretory inhibitor of leukocyte proteinase, cytokines, $C$-reactive protein, alanine aminotransferase, aspartate aminotransferase, liver, community-acquired pneumonia in children.

\section{Introduction}

Intensification of protective inflammatory reactions in children with pneumonia may occur in response to the invasion of pathogens, which is observed in the interaction of receptors on the surface of cell membranes with immune complexes [7, 13, 16]. In this case, the inflammatory process that develops in the lower respiratory tract is accompanied by the release from cells and activation of proteases - extracellular matrix metalloproteases and serine proteases $[1,15]$. Normally, these proteases are synthesized in cells and accumulate in the primary lysosomes of polymorphonuclear leukocytes [3]. They play an important role in the degradation of the components of the extracellular matrix, and thus determine the prognosis and nature of the pathological process in the body [4]. Regulation of lysosomal protease activity is carried out by local and/or systemic inhibitors, which are able to prevent 
excessive proteolysis [12]. As a result, tissue destruction is prevented by an excessive immune response. Such antiproteases include the secretory leukocyte pro tease inhibitor, SLPI [17]. It is known [2,5] that this inhibitor is produced by epithelial cells of the upper and lower respiratory tract, as well as macrophages and neutrophils of mucous membranes. As a component of local immunity, SLPI can act as an important regulator of innate and acquired immunity and provide control of the local inflammatory response [14].

Because SLPI has not only antiprotease (C-terminal domain) but also antibacterial (N-terminal domain) action [9], determining its level in the serum of children with community-acquired pneumonia is an important factor in the mechanism of disease development. The study of SLPI levels in children with pneumonia is important because it may indicate a violation of protease-antiprotease balance and predict local regulation of proteolytic activity at different degrees of severity of pneumonia in children. The effects of hepatobiliary lesions on the plasma SLPI levels of children with pneumonia, as well as the relationship between SLPI levels and markers of the infectiousinflammatory process, interleukins and $\mathrm{C}$-reactive protein (CRP), remain unclear.

The aim of the study was to assess the level of SLPI as a marker of pro-inflammatory response in the serum of young children, depending on the severity of pneumonia, including the presence or absence of lesions of the hepatobiliary system.

\section{Materials and methods}

We examined 338 children with community-acquired pneumonia, aged 1 to 36 months, who were hospitalized in the Infectious Diseases Boxing Department for Young Children of the Vinnytsia Regional Children's Clinical Hospital.

Verification of the diagnosis of community-acquired pneumonia in young children was performed according to the recommendations of the British Thoracic Society (British Thoracic Society guidelines for the management of community acquired pneumonia in children: update 2011). Pre-antibacterial treatment was not performed in patients prior to admission to the hospital.

In the presence or absence of lesions of the hepatobiliary system, all patients were divided into two groups: Group I - 186 children who had pneumonia and HBS lesions; Group II - 152 patients with pneumonia without damage to the hepatobiliary system. The comparison group included 40 healthy children.

In each group, the results were analyzed depending on the severity of pneumonia (moderate, severe), age (1-12 months and 13-36 months) and sex (boys, girls).

The level of SLPI in the serum was determined by enzyme-linked immunosorbent assay using a diagnostic test system from "IMMUNOTECH" (France).

"IBM SPSS Statistica" Version 12 (20) was used for statistical processing of results using parametric and nonparametric methods.

\section{Results}

The results of the studies showed that all patients had an increase in serum SLPI level (table 1). Thus, in children with pneumonia and lesions of the hepatobiliary system, the level of SLPI was 4.94 times higher than in healthy children $(p<0.05)$. Similar changes were observed in patients with pneumonia without lesions of the hepatobiliary system, in whom the SLPI was 5.45 times higher than in healthy children $(p<0.05)$ (see table 1 ).

In patients with hepatic impairment, SLPI levels were elevated in both moderate and severe pneumonia. However, if with moderate pneumonia the level of SLPI was $807.8 \pm 54.2 \mathrm{pg} / \mathrm{ml}$, which is 3.49 times higher than the comparison group $(231.3 \pm 13.7 \mathrm{pg} / \mathrm{ml})(p<0.05)$, then with severe - the level of SLPI was $1496 \pm 175 \mathrm{pg} / \mathrm{ml}$, which is 6.47 times higher than the comparison group $(p<0.05)$ (Table 2). Apparently, in severe pneumonia, destructive processes of the extracellular matrix involving proteolytic enzymes occur in the lung tissue to a greater extent, in response to which appropriate protease inhibitors, in particular SLPI, are synthesized [4]. This reduces the detoxification function of the liver.

In patients without liver damage, the level of SLPI in the

Table 1. SLPI content in children with community-acquired pneumonia depending on the presence of lesions of the hepatobiliary system $(\mathrm{M} \pm \mathrm{m})$.

\begin{tabular}{|l|c|c|c|}
\hline \multirow{2}{*}{ Serum content } & \multicolumn{2}{|c|}{ All patients, $\mathrm{n}=338$} & \multirow{2}{*}{$\begin{array}{c}\text { Healthy } \\
\text { children, } \mathrm{n}=40\end{array}$} \\
\cline { 2 - 3 } & $\begin{array}{c}\text { with HBS } \\
\text { lesions }\end{array}$ & $\begin{array}{c}\text { without HBS } \\
\text { lesions }\end{array}$ & \\
\hline $\mathrm{SLPI}(\mathrm{pg} / \mathrm{ml})$ & $1144 \pm 51^{*}$ & $1261 \pm 42^{*}$ & $231.3 \pm 13.7$ \\
\hline
\end{tabular}

Notes: * - statistically significant difference compared to healthy children, $\mathrm{p}<0.05$; HBS - hepatobiliary system.

Table 2. SLPI content in children with pneumonia, depending on the severity of the disease $(\mathrm{M} \pm \mathrm{m})$.

\begin{tabular}{|c|c|c|c|c|c|}
\hline \multirow{3}{*}{ Indicator } & \multicolumn{4}{|c|}{ All patients, $\mathrm{n}=338$} & \multirow{2}{*}{$\begin{array}{c}\text { Healthy } \\
\text { children, } \\
n\end{array}$} \\
\cline { 2 - 5 } & with HBS lesions & \multicolumn{2}{|c|}{ without HBS lesions } \\
\cline { 2 - 4 } & moderate & severe & moderate & severe & \\
\hline $\begin{array}{c}\mathrm{SLPI} \\
(\mathrm{pg} / \mathrm{ml})\end{array}$ & $807.8 \pm 54.2^{\star}$ & $1496 \pm 175^{\star}$ & $1172 \pm 63^{\star}$ & $1303 \pm 46^{\star}$ & $231.3 \pm 13.7$ \\
\hline
\end{tabular}

Note: * - statistically significant difference compared to healthy children, $\mathrm{p}<0.05$; HBS - hepatobiliary system.

Table 3. SLPI content in children with community-acquired pneumonia, depending on age, $(\mathrm{M} \pm \mathrm{m})$.

\begin{tabular}{|c|c|c|c|c|c|}
\hline \multirow{4}{*}{ Indicator } & \multicolumn{3}{|c|}{ All patients, $\mathrm{n}=338$} & \multirow{2}{*}{$\begin{array}{c}\text { Healthy } \\
\text { children, } \\
\mathrm{n}=40\end{array}$} \\
\cline { 2 - 5 } & age 1-12 month & \multicolumn{2}{|c|}{ age 13-36 month } \\
\cline { 2 - 5 } & $\begin{array}{c}\text { with HBS } \\
\text { lesions }\end{array}$ & $\begin{array}{c}\text { without } \\
\text { HBS } \\
\text { lesions }\end{array}$ & $\begin{array}{c}\text { with HBS } \\
\text { lesions }\end{array}$ & $\begin{array}{c}\text { without HBS } \\
\text { lesions }\end{array}$ & \\
\hline $\begin{array}{c}\mathrm{SLPI} \\
(\mathrm{pg} / \mathrm{ml})\end{array}$ & $1270 \pm 61^{*}$ & $1388 \pm 46^{*}$ & $841.5 \pm 85.9^{*}$ & $782.4 \pm 69.4^{*}$ & $231.3 \pm 13.7$ \\
\hline
\end{tabular}

Note: * - statistically significant difference compared with healthy children, $\mathrm{p}<0.05$; GBS - hepatobiliary system. 
Table 4. Gender-based SLPI in children with community-acquired pneumonia, (M $\pm m)$.

\begin{tabular}{|l|c|c|c|c|c|c|}
\hline \multirow{3}{*}{ Indicator } & \multicolumn{4}{|c|}{ All patients, $\mathrm{n}=338$} & \multicolumn{3}{c|}{ Healthy children, $\mathrm{n}=40$} \\
\cline { 2 - 6 } & \multicolumn{2}{|c|}{ with HBS lesions } & \multicolumn{2}{c|}{ without HBS lesions } & \multicolumn{3}{c|}{} \\
\cline { 2 - 7 } & boys, $\mathrm{n}=103$ & girls, $\mathrm{n}=87$ & boys, $\mathrm{n}=69$ & girls, $\mathrm{n}=79$ & boys, $\mathrm{n}=21$ & girls, $\mathrm{n}=19$ \\
\hline $\mathrm{SLPI}(\mathrm{pg} / \mathrm{ml})$ & $1095 \pm 61^{*}$ & $1197 \pm 85^{*}$ & $1333 \pm 64^{*}$ & $1231 \pm 57^{*}$ & $227.6 \pm 30.8$ & $254.3 \pm 23.1$ \\
\hline
\end{tabular}

Note: * - statistically significant difference compared to healthy children, $p<0.05$; GBS - hepatobiliary system.

blood was equally increased in both moderate and severe community-acquired pneumonia. It was found that in moderate pneumonia the level of SLPI increased 5.07 times, and in severe - 5.63 times compared with healthy children $(p<0.05)$ (see Table 2). The established fact indicates that in community-acquired pneumonia the liver plays a major role in protecting the child's body from pathogens that provoke infectious and inflammatory processes in the lungs. The issues of protection of the body of children of different age groups remain open, because at an early age the adaptive-protective systems are not yet sufficiently developed.

The results of determining the level of SLPI in the serum of children with community-acquired pneumonia, which we divided by age showed that in children aged 1-12 months, its level significantly exceeded not only control, but also children aged 13-36 months (Table 3 ). Thus, in children with pneumonia aged 1-12 months with liver damage, the SLPI level was $1270 \pm 61 \mathrm{pg} / \mathrm{ml}$, and without liver damage $1388 \pm 46 \mathrm{pg} / \mathrm{ml}$, which is 5.49 times and 6.0 times higher than healthy children, respectively $(p<0.05)$ (see Table 3 ).

At the same time, in children aged 13-16 months the studied values were lower: $841.5 \pm 85.9 \mathrm{pg} / \mathrm{ml}$ - in children with community-acquired pneumonia with liver damage and $782.4 \pm 69.4 \mathrm{pg} / \mathrm{ml}$ - in children with pneumonia without liver damage, which is 3.64 times and 3.38 times higher than healthy children, respectively $(p<0.05)$ (see Table 3 ).

Analysis of the results of the study of the level of SLPI depending on gender showed that the difference between the studied indicator in boys and girls was not found. In children with hepatobiliary disorders, the SLPI level was $1095 \pm 61 \mathrm{pg} / \mathrm{ml}$ in boys and $1197 \pm 85 \mathrm{pg} / \mathrm{ml}$ in girls (Table 4). Similar changes were observed in patients without hepatobiliary system damage, in whom SLPI was 1333 \pm $64 \mathrm{pg} / \mathrm{ml}$ in boys and $1231 \pm 57 \mathrm{pg} / \mathrm{ml}$ in girls (see Table 4). However, it should be noted that in all patients without liver damage (boys and girls) SLPI levels were higher than in patients with hepatobiliary disorders.

The second stage of the study was the correlation between the level of SLPI in the serum and the indicators of infectious-inflammatory process - IL-1, IL-6 and CRP. The analysis showed that the highest positive correlation was observed between the level of SLPI and the level of IL1 in the blood with a value of $r_{x y}=+0.179$, which indicates the fact that SLPI production increases under the influence of IL-1 [8]. The correlation between the level of SLPI and the level of IL- 6 in the blood was $r_{x y}=+0.127$, which indicates a lower direct relationship between these indicators. The correlation coefficient between the content of SLPI and CRP in blood serum is equal to $r_{x y}=-0.215$.

Studies have shown an association between the level of SLPI indicators of cytolysis syndrome - ALT, AST and GTP in the blood. Thus, by calculating the Pearson correlation coefficient, a positive correlation was found between the level of SLPI and ALT activity with the level of $r_{x y}=+0.130$ and a negative correlation between the level of SLPI and AST and GTP. Moreover, the correlation coefficient is lower for the GTP $-r_{x y}=-0.215$ compared with AST $r_{x y}=-0.054$.

The results of the analysis of the relationship between total bilirubin and alkaline phosphatase activity showed a weak correlation with the level of SLPI, which is equal to $r_{x y}=+0.047$ and $r_{x y}=+0.025$, respectively, which is apparently related to the synthesis of this inhibitor by gallbladder epithelial cells conditions of damage to the hepatobiliary system.

\section{Discussion}

The development of infectious and inflammatory processes in the lungs is accompanied by the release and activation of a large family of proteases that determine the nature of the disease, as they play an important role in the degradation of extracellular matrix components [6]. Maintaining the integrity of the target organ is provided by inhibitors of protease activity that prevent excessive proteolysis, among which SLPI is one of the most important inhibitors of proteases that function in the respiratory tract. Therefore, determining the level of SLPI in the serum of young children will predict the course of pneumonia in the presence or absence of lesions of the hepatobiliary system.

SLPI production in patients with pneumonia may increase under the influence of $\mathrm{IL}-1$, tumor necrosis factor- $\alpha$, epidermal growth factor, neutrophil elastase [8]. On the other hand, increased levels of SLPI in inflammatory processes in lung tissue may occur in response to activation of proteolytic enzymes in children with community-acquired pneumonia [17], indicating the activation of compensatory responses in children. Increasing the level of SLPI helps to suppress the action of neutrophil elastase, trypsin and cathepsin $\mathrm{G}$ (to a lesser extent) [8]. It should be noted that in patients with lesions of the hepatobiliary system, the increase in SLPI is less than in patients without liver damage. This fact can be explained by the fact that SLPI is partially synthesized in the liver [10], so when this organ is affected, the level of SLPI in the bloodstream decreases, which will negatively affect the state of the child's body. In 
addition, changes in the level of SLPI in the blood may depend on the intensity of the inflammatory process in the lungs, which makes it necessary to study the level of SLPI depending on the severity of pneumonia.

Since the studied protease inhibitor may also affect the regulation of anti-inflammatory cytokines IL-10 and transforming growth factor macrophages [11], a sharp increase in children aged 1-12 months indicates the activation of the body's defense systems. In addition, SLPI can induce neutrophil chemotaxis, reduce their death by apoptosis and does not significantly affect the release of IL-8, so such a sharp increase (response) of this inhibitor can be used to assess the condition of sick children and chronic pathological process [4]. At the same time, this will determine the tactics of treatment in these groups of patients.

As a result of our research, it was found that with the development of community-acquired pneumonia in young children in the body increases the level of SLPI with higher rates in severe disease. In patients with lesions of the hepatobiliary system, this increase is less pronounced, which indicates lower protection of the body from destructive processes in the infectious-inflammatory process. The level of SLPI does not depend on gender, but is higher in children aged 1-12 months, compared with the group of children 13-36 months. The results indicate that the liver plays an important role in protecting the body from an infectious agent that is tropical to the tissues of the respiratory system, indicating the need for hepatoprotectors for patients with hepatobiliary diseases, which can facilitate the course and prevent complications of infectiousinflammatory process in these patients.

Analysis of the correlations between serum SLPI levels and infectious-inflammatory indicators suggests that increased CRP in the blood of patients with pneumonia may indicate destructive changes in the liver, resulting in decreased SLPI levels. Because the liver is part of the hepatobiliary system, along with liver dysfunction, the work of the gallbladder may be impaired under conditions of infectious-inflammatory process in pneumonia. Therefore, we determined the correlation coefficient between the level of SLPI and cytolysis and cholestasis.

The negative correlation between the level of SLPI and the activity of AST and GTP enzymes in the blood indicates that an increase in the level of SLPI in the blood is accompanied by a decrease in the activity of AST and GTP. This may indicate destructive processes in the liver and increased inflammatory processes in the lungs with

\section{References}

[1] Bradshaw, J. L., Caballero, A. R., Bierdeman, M. A., Adams, K. V., Pipkins, H. R., Tang, A., ... \& McDaniel, L. S. (2018). Pseudomonas aeruginosa protease IV exacerbates pneumococcal pneumonia and systemic disease. Msphere, 3(3), e00212-18. doi: 10.1128/mSphere.00212-18

[2] Camper, N., Glasgow, A. M. A., Osbourn, M., Quinn, D. J., Small, D. M., McLean, D. T., ... \& Taggart, C. C. (2016). A secretory activation of proteolytic activity, destruction of cells in the lungs, in response to which the level of SLPI increases, ie triggers a focal inflammatory response.

Thus, the results show that the importance of protease inhibitors in the regulation of intracellular proteolysis in the development of pathological processes in lung tissue remains important for predicting the prognosis of the disease. There is a significant correlation between the influence of cytokines (inflammatory mediators) and protective factors (protease inhibitors) on the development and maintenance of inflammatory processes in the lungs in community-acquired pneumonia, the relationship with the severity of the disease, the development of hepatobiliary pathology.

\section{Conclusion}

1. Determination of the level of protease inhibitor in the serum of young children with community-acquired pneumonia showed that the disease is accompanied by a risk of extracellular damage in lung tissue, in response to increased SLPI production (in children with hepatobiliary system, SLPI level 4.94 times patients without lesions of the hepatobiliary system - 5.45 times higher than healthy children). Plasma SLPI levels decrease with hepatobiliary damage.

2. In patients with hepatic impairment, the level of SLPI increased in both moderate and severe pneumonia ( 3.49 and 6.47 times, respectively) compared with the control group. In patients with pneumonia without liver damage, the level of SLPI in the blood was equally increased in both moderate and severe nosocomial pneumonia (5.07 and 5.63 times, respectively) compared with healthy children.

3. In children aged 1-12 months with pneumonia and hepatobiliary lesions, the SLPI level was 5.49 times, and without hepatobiliary lesions - 6.0 times higher than in the control group. In children 13-36 months, the studied rate was 3.64 times higher than pneumonia with lesions of the hepatobiliary system and 3.38 times (without lesions of the hepatobiliary system) was higher than in healthy children.

4. The level of SLPI in the serum correlates with the content of pro-inflammatory cytokines, which indicates the unidirectional changes in the formation of local immunity in response to lung tissue damage $(r x y=+0.127-(+0.179)$, in addition, there is a negative relationship between its content and activity of AST, GTP and CRP at the level of $r x y=-0.054$ $(-0.215)$, which indicates liver dysfunction with the development of infectious-inflammatory process in the body.

leukocyte protease inhibitor variant with improved activity against lung infection. Mucosal immunology, 9(3), 669-676. doi: 10.1038/mi.2015.90

[3] Chiang, T. Y., Tsao, S. M., Yeh, C. B., \& Yang, S. F. (2014). Matrix metalloproteinases in pneumonia. Clinica Chimica Acta, 433, 272-277. doi: 10.1016/j.cca.2014.03.031

[4] Dvorak, J., \& Horn, M. (2018). Serine proteases in schistosomes 
and other trematodes. International journal for parasitology, 48(5), 333-344. doi: 10.1016/j.ijpara.2018.01.001

[5] Habgood, A. N., Tatler, A. L., Porte, J., Wahl, S. M., Laurent, G. J., John, A. E., ... \& Jenkins, G. (2016). Secretory leukocyte protease inhibitor gene deletion alters bleomycin-induced lung injury, but not development of pulmonary fibrosis. Laboratory investigation, 96(6), 623-631. doi: 10.1038/labinvest.2016.40

[6] Jin, Y., Li, Y., Wang, X., \& Yang, Y. (2019). Secretory leukocyte protease inhibitor suppresses HPV E6-expressing HNSCC progression by mediating NF-kB and Akt pathways. Cancer cell international, 19(1), 1-19. doi: 10.1186/s12935-019-09427

[7] Leung, A. K., Wong, A. H., \& Hon, K. L. (2018). Communityacquired pneumonia in children. Recent patents on inflammation \& allergy drug discovery, 12(2), 136-144. doi: $10.2174 / 1872213 \times 12666180621163821$

[8] Liu, L., Ma, Z., Sun, W. W., \& Cao, J. P. (2018). Upregulated expression of secretory leukocyte protease inhibitor in lung by inhalation of high concentration of sulfur dioxide. Chinese medical journal, 131(16), 2005-2007. doi: 10.4103/03666999.238143

[9] Nugteren, S., \& Samsom, J. N. (2021). Secretory Leukocyte Protease Inhibitor (SLPI) in mucosal tissues: Protects against inflammation, but promotes cancer. Cytokine \& Growth Factor Reviews, (59), 22-35. doi: 10.1016/j.cytogfr.2021.01.005

[10] Nugteren, S., Goos, J. A., Delis-van Diemen, P. M., SimonsOosterhuis, Y., Lindenbergh-Kortleve, D. J., van Haaften, D. H., ... \& Samsom, J. N. (2020). Expression of the immune modulator secretory leukocyte protease inhibitor (SLPI) in colorectal cancer liver metastases and matched primary tumors is associated with a poorer prognosis. Oncoimmunology, 9(1), 1832761. doi: 10.1080/2162402X.2020.1832761

[11] Reitman, S., \& Frankel, S. (1957). Acolorimetric method for the determination of serum glutamic oxalacetic and glutamic pyruvic transaminases. American journal of clinical pathology, 28(1), 56-63. doi: 10.1093/ajcp/28.1.56

[12] Samejima, T., Nagamatsu, T., Akiba, N., Fujii, T., Sayama, S., Kawana, K., ... \& Fujii, T. (2021). Secretory leukocyte protease inhibitor and progranulin as possible regulators of cervical remodeling in pregnancy. Journal of Reproductive Immunology, 143, 103241. doi: 10.1016/j.jri.2020.103241

[13] Satici, C., Demirkol, M. A., Altunok, E. S., Gursoy, B., Alkan, M., Kamat, S., ... \& Esatoglu, S. N. (2020). Performance of pneumonia severity index and CURB-65 in predicting 30-day mortality in patients with COVID-19. International Journal of Infectious Diseases, 98, 84-89. doi: 10.1016/j.ijid.2020.06.038

[14] Tang, R., Botchway, B. O., Meng, Y., Zhang, Y., Zhou, C., Jiang, J., \& Liu, X. (2020). The inhibition of inflammatory signaling pathway by secretory leukocyte protease inhibitor can improve spinal cord injury. Cellular and molecular neurobiology, 40(7), 1067-1073. doi: 10.1007/s10571-020-00799-1

[15] Van Den Boogaard, F. E., Brands, X., Duitman, J., De Stoppelaar, S. F., Borensztajn, K. S., Roelofs, J. J., ... \& van der Poll, T. (2018). Protease-activated receptor 2 facilitates bacterial dissemination in pneumococcal pneumonia. The Journal of infectious diseases, 217(9), 1462-1471. doi: 10.1093/infdis/ jiy010

[16] Yun, K. W., Wallihan, R., Juergensen, A., Mejias, A., \& Ramilo, O. (2019). Community-acquired pneumonia in children: myths and facts. American journal of perinatology, 36(S 02), S54S57. doi: 10.1055/s-0039-1691801

[17] Zhong, Q. Q., Wang, X., Li, Y. F., Peng, L. J., \& Jiang, Z. S. (2017). Secretory leukocyte protease inhibitor promising protective roles in obesity-associated atherosclerosis. Experimental Biology and Medicine, 242(3), 250-257. doi: 10.1177/1535370216672747 